\title{
Particle acceleration at the discontinuous flow boundary of collimated cylindrical jets
}

\author{
Stephen O’Sullivan, ${ }^{a, *}$ Andrew M. Taylor ${ }^{b}$ and Brian Reville ${ }^{c}$ \\ ${ }^{a} T U$ Dublin, \\ School of Mathematical Sciences, Grangegorman Lower, Dublin 7, Ireland \\ ${ }^{b} \mathrm{DESY}$, \\ D-15738 Zeuthen, Germany \\ ${ }^{c} M P I K$, \\ Saupfercheckweg 1, Heidelberg, Germany \\ E-mail: stephen.osullivan@TUDublin.ie
}

We revisit the issue of particle acceleration at the interface between a collimated laminar jet and a static ambient medium. The contrast between standard diffusive scattering treatments and anomalous transport in synthetic field constructions is explored. A particular emphasis is placed on the necessity for physically consistent particle transport considerations. The temporal, spatial and spectral features of the process are discussed, in the context of potential UHECR production, as well as further observational consequences.

\footnotetext{
*** 37th International Cosmic Ray Conference (ICRC2021), ***

*** 12-23 July 2021 ***

*** Berlin, Germany - Online ***
}

\footnotetext{
${ }^{*}$ Presenter
} 


\section{Introduction}

It is now firmly established that continuous acceleration of energetic particles is required to account for multi-wavelength observations of extra-galactic jets, both in X-ray [1] and gammaray [2]. The physical process underlying this energisation remains an open question, though it must be able to overcome the relatively short cooling times, demanding that a fast acceleration mechanism is at play. A promising candidate in this regard is the shear acceleration mechanism [3], whereby particles gain energy due to repeated scattering on magnetic waves/inhomogeneities that are advected with the non-uniform flow.

Since shear acceleration is a second-order Fermi process, it becomes increasingly effective as the relative flow velocities increase, particularly for the case of sharp gradients in the velocity flow profile. To see this, consider the idealised case of a cylindrical jet with symmetric axial flow profile $\left.\boldsymbol{\beta}(r)=\frac{1}{2} \beta_{\text {jet }}\left[1-\tanh \left(\left(r-r_{\text {jet }}\right) / L\right)\right)\right] \hat{z}$, where $\beta_{\text {jet }}$ is the jet speed on the axis in units of $c$, and $L$ determines the thickness of the shear layer. For particles with Larmor radius $r_{\mathrm{g}} \ll L$, the shear layer is smooth, and the acceleration time is well known [e.g. 4]

$$
\frac{c t_{\mathrm{acc}}}{r_{\mathrm{g}}} \approx \frac{15}{4+\alpha} \frac{r_{\mathrm{g}}}{c t_{\mathrm{scat}}^{\prime}} \frac{\left(L / r_{\mathrm{g}}\right)^{2}}{\beta_{\text {jet }}^{2}}\left[1+4\left(\frac{c t_{\mathrm{scat}}^{\prime}}{r_{\mathrm{g}}}\right)^{2}\right] .
$$

Here $t_{\text {scat }}^{\prime} \propto \gamma^{\alpha}$ is the scattering time in the local fluid frame, with $\gamma$ the Lorentz factor of the accelerating particles. The term in square brackets on the right-hand side is due to the guide field, which, being frozen into a shearing flow, should only have components in the $z-\theta$ plane, as large scale components in the radial direction will be sheared. Thus, unless the mean free path is less than the gyro-radius, i.e. the particles are unmagnetised, the ability of particles to sample the large velocity gradients is inhibited, and acceleration is slow relative to its gyration period (i.e substantially sub-Bohm).

In the opposite limit, $r_{\mathrm{g}} \gg L$, particles effectively see the shear layer as a discontinuity. In this case, to a good approximation, we can assume that the particle energy is conserved while scattering in the local frame. Performing Lorentz transformations at each crossing, and assuming $\gamma \gg \Gamma_{\text {jet }}$, where $\Gamma_{\text {jet }}=\left(1-\beta_{\text {jet }}^{2}\right)^{-1 / 2}$ is the jet Lorentz factor on the axis, we can easily calculate the energy jump per cycle as a function of the crossing angles. Defining $\mu_{1(2)}=\cos \theta_{1(2)}$ as the pitch angle of in-going (out-going) particles with respect to the $z$-axis, measured on crossing the surface $r=r_{\text {jet }}$, the ratio of energies is

$$
\frac{E_{\text {final }}}{E_{\text {init }}}=\frac{1}{\Gamma_{\text {jet }}^{2}\left(1+\beta_{\text {jet }} \mu_{1}^{\prime}\right)\left(1-\beta_{\text {jet }} \mu_{2}\right)} .
$$

Here, the initial and final energies are measured in the "ambient" frame $\left(r>r_{\text {jet }}\right)$, and primed quantities are measured in the "jet" frame. If particles are isotropised in each region before crossing back in to the other side of the shear boundary, relative to an observer in the opposite frame, the incoming particles will appear like a beam i.e. $\mu_{2} \approx-\mu_{1}^{\prime} \approx \beta_{\text {jet }}$. Energy boosts as large as $4 \Gamma_{\text {jet }}^{2}$ are in theory achievable. Thus, for ultra-relativistic jets, the per-cycle acceleration due to crossing the shear layer can be substantial. However, the assumption of isotropisation limits the cycle time to be at least as long as the isotropisation time. In practice, the acceleration time is determined by competition between these two effects, namely the mean cycle time and the associated 
mean energy kick per cycle. As with Fermi acceleration at ultra-relativistic shocks [5], we will find that the mean energy kick per cycle is of order unity.

To verify this claim, and to develop a deeper understanding of the relevant physical constraints, we have performed a numerical analysis of discontinuous shear using Monte-Carlo simulations. Previous numerical investigations into the process have, to our knowledge, exclusively relied on random scattering approaches i.e. particles are scattered in angle according to some prescription, their energy being conserved in the local fluid frame in each scattering [e.g. 6]. As we show in the following discussion, this tends to be a gross simplification of the problem, and overlooks geometrical constraints on the field structure in the vicinity of the shear layer boundary. These constraints turn out to be critical for the particle transport close to the shear layer, and result in significantly different scaling to the acceleration times than is implied by a naive application of the random scattering approach.

\section{Methods}

The preferred approach to the simulation of turbulent fields in the literature relies on the superposition of Fourier plane modes [7]. In the context of sheared flows, in which the inner and outer volumes must move relative to one another, this raises considerations of solenoidality which require particular attention. In this work, we expand on the adoption of Bessel modes for the representation of fields in cylindrical jets by previous authors [e.g. 8].

A natural consequence of our solenoidal field prescription is dependency of the local field correlation scale on the distance from the shear discontinuity. This effectively suppresses fluctuations in the field at the scale of the distance from the boundary. The role of different kinetic and fluid instabilities on the jet interface is an open question [9, 10], but the collimation out to several kiloparsecs observed in many high-power jets generally indicate that stability can be maintained over large distances. We note that scaling laws for turbulence suppression in shearing flows have been studied by various authors as a mechanism for the stabilization of turbulence in tokamak plasmas [11-13].

Motivated by this discussion, radial dependency of the magnetic field correlation scale in the region of the shear discontinuity is modeled via the ansatz

$$
l_{\mathrm{c}}(r) \sim \begin{cases}|\Delta r|, & |\Delta r|<l_{\mathrm{c}}^{0}, \\ l_{\mathrm{c}}^{0}, & |\Delta r| \geq l_{\mathrm{c}}^{0},\end{cases}
$$

where $\Delta r \equiv r-r_{\text {jet. }}$ Assuming an isotropic power spectrum $\left\langle B^{2}\right\rangle(r)=\int_{0}^{\lambda_{\max }} S(\lambda) \mathrm{d} \lambda$ specified by the power-law $S(k)=A \lambda^{q}$ yields a representative field variance

$$
\left\langle B^{2}\right\rangle \sim \begin{cases}B_{0}^{2}\left(\frac{|\Delta r|}{l_{\mathrm{c}}^{0}}\right)^{q-1}, & |\Delta r|<l_{\mathrm{c}}^{0}, \\ B_{0}^{2} \frac{r_{\mathrm{jet}}}{r}, & |\Delta r| \geq l_{\mathrm{c}}^{0},\end{cases}
$$

where $A$ is a fixed normalisation constant determined by $B_{0}$. Figure 1 confirms that this scaling holds in the simulated fields for Kolmogorov turbulence with $q=5 / 3$.

Particle motions within the synthetic field are obtained via a new Boris-type integrator which properly conserves phase space volume (and hence has excellent energy conservation properties) 


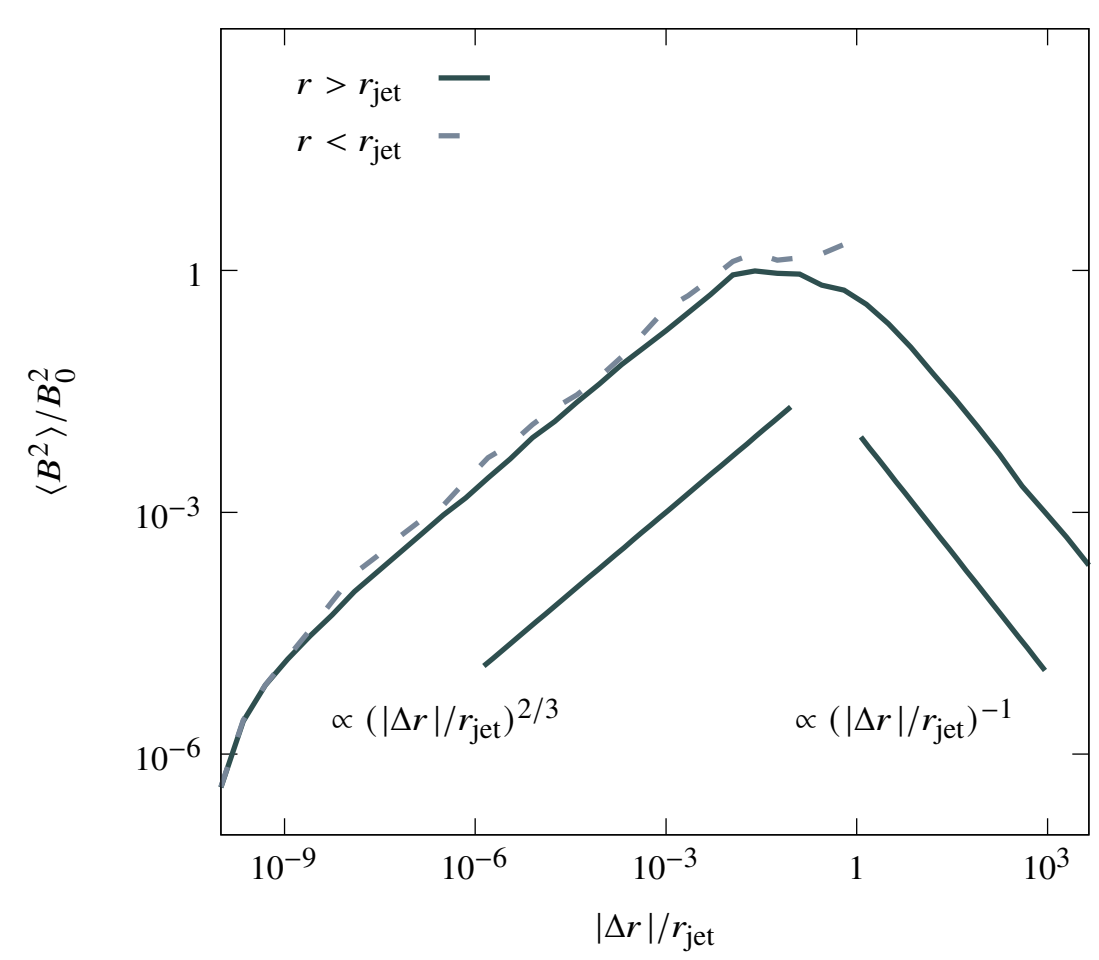

Figure 1: Magnetic field power as a function of displacement from shear discontinuity. The solid/dashed line indicates the power on the exterior/interior of the jet. Guidelines scale as $q-1=2 / 3$ for Kolmogorov turbulence within $\sim l_{\mathrm{c}}$ of the discontinuity, and -1 otherwise.

and operates efficiently over a large dynamic range. Additionally, it does not suffer from the fictitious forces identified by Vay in studies of the conventional Boris solver [14]. A sample trajectory is shown in Figure 2.

\section{Results}

In order to understand the results from simulated particle motions, it is helpful to first consider the return frequency distribution function obtained from large ensembles. This distribution function records the binned return frequency over a single cycle. Particles are injected isotropically on the interior of the jet, with the energy such that a prescribed reference value would be realised on perpendicular exit into the ambient medium. An initial particle energy delta distribution is ensured by resetting the particles to the reference value on their initial crossing into the exterior volume. From this point, a complete acceleration cycle for a given particle is measured between the two subsequent times of entry into the jet. We find this approach effective in capturing the behaviour of an adequately relaxed population of monoenergetic particles. Along with return frequency distribution, insight is also provided by considering the corresponding log-energy boost distribution. From these two distribution functions, the resultant acceleration rate (i.e. inverse of the acceleration time) can be both derived and easily understood. 


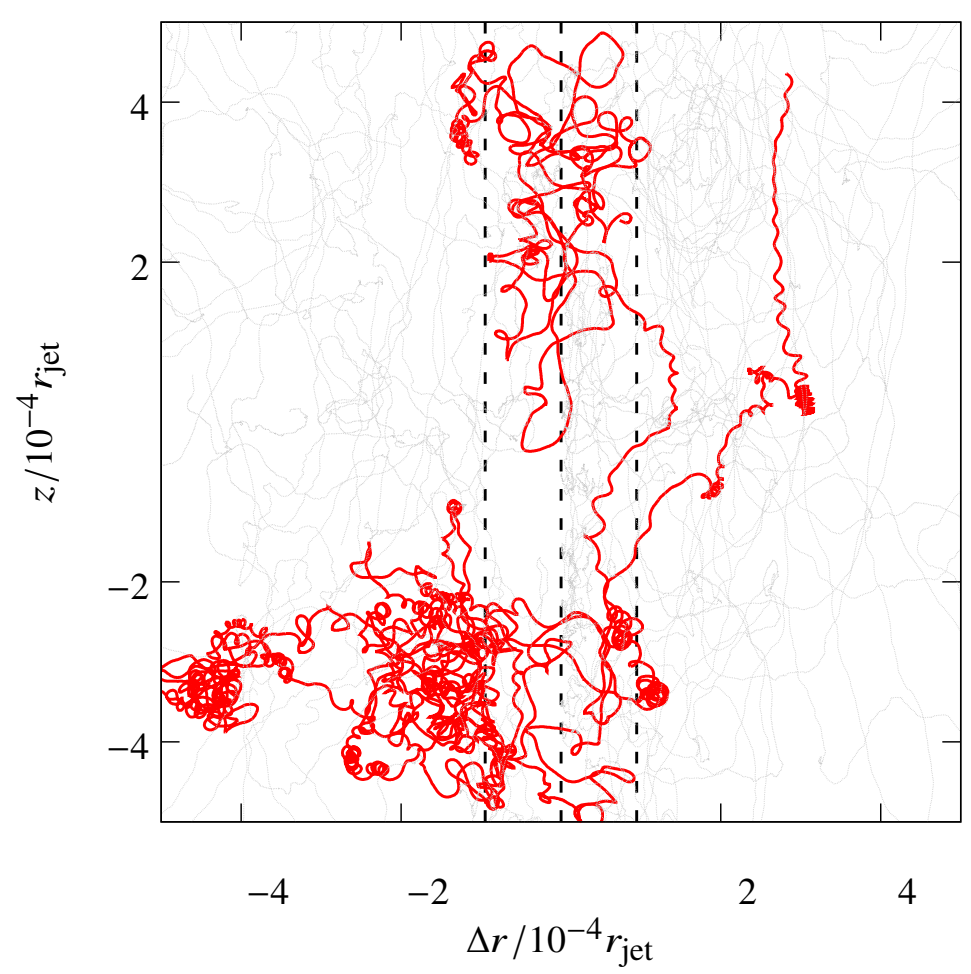

Figure 2: Sample field line integration in the region of a discontinuity for a particle with reference rigidity $\rho=10^{-6}$. The dashed vertical lines indicate the rectilinear range for the mean acceleration cycle time about the discontinuity. A sample set of projected field lines is shown in the background. Note that no acceleration is taking place for this illustration (i.e. $\Gamma_{\text {jet }}=1$ ).

Examples of these two distribution functions obtained are shown in Fig. 3, for both the "random" and "field" cases. A shear flow with $\Gamma_{\text {jet }}=10$, and a turbulent power spectrum with Kolmogorov scaling has been adopted in both instances.

In order to obtain the acceleration times from these distribution functions, the mean acceleration frequency is calculated using

$$
\left\langle f_{\mathrm{acc}}\right\rangle=\frac{\int \Delta_{\ln E} f \frac{\mathrm{d} N}{\mathrm{~d} f} \mathrm{~d} f}{\int \frac{\mathrm{d} N}{\mathrm{~d} f} \mathrm{~d} f} .
$$

The acceleration time is then defined as $t_{\text {acc }}=1 /\left\langle f_{\text {acc }}\right\rangle$.

In Fig. 3, the acceleration time determined from our simulations for each rigidity particle group is indicated as a vertical dashed line (with the same corresponding line colour as that for the rigidity group it is applicable for). Rigidity here is defined by $\rho \equiv r_{\mathrm{g}} / r_{\text {jet }}$, where $r_{\mathrm{g}}=E / e B_{0}$ is the gyro-radius of a particle with energy $E$ in the reference field $B_{0}$. As appreciated from both the "random" and "field" distribution function results shown in Fig. 3, the acceleration time is strongly dictated by the return time distribution function. Furthermore, the faster growth of the acceleration time with rigidity for the "field" case compared to the "random" case can be directly appreciated from the spacing of the vertical dashed lines. 

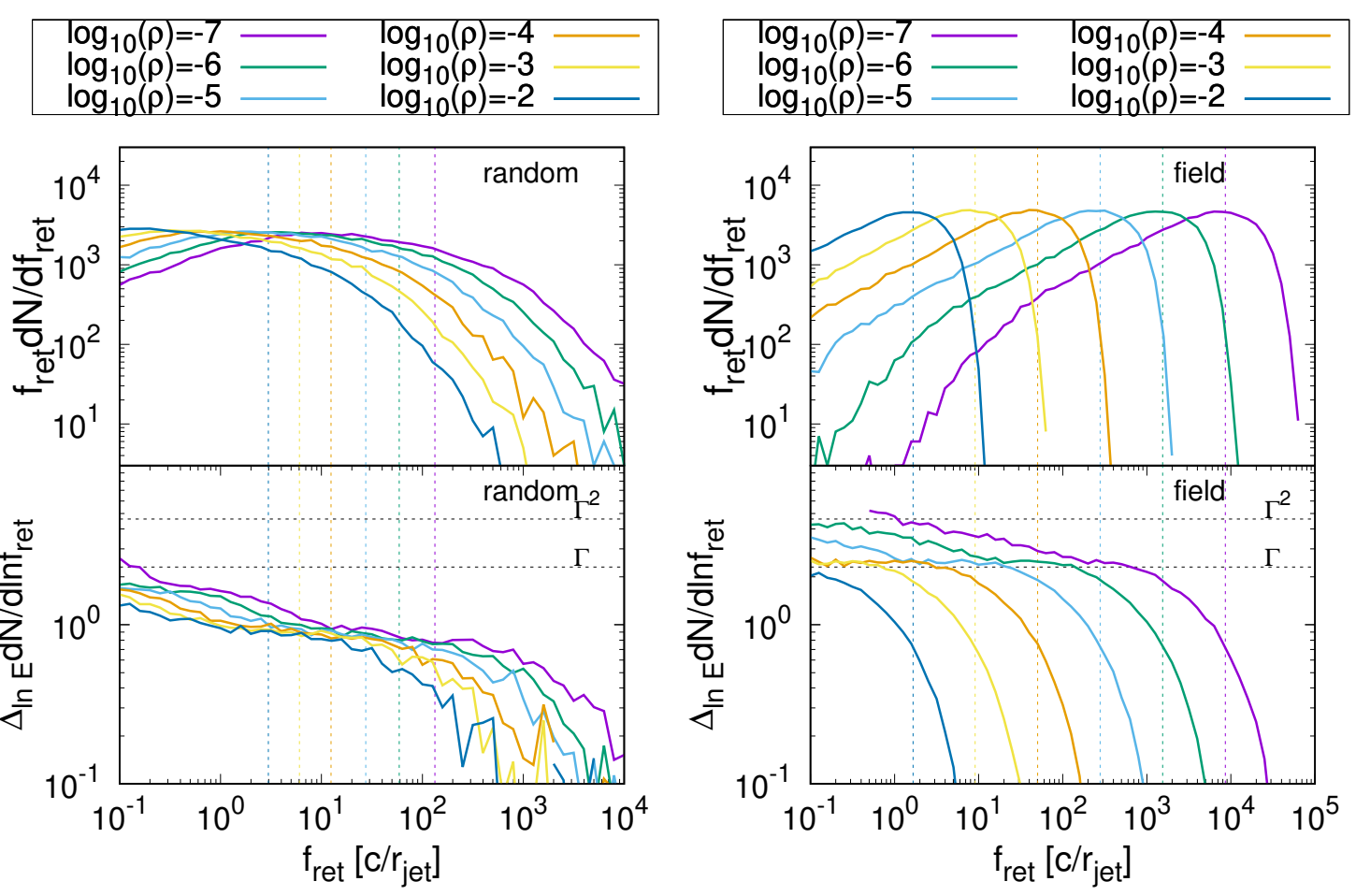

Figure 3: [Left] The top-panel shows the return frequency distribution obtained from a large ensemble of particles whose scattering times are dictated by a simple scattering function, which we label "random". The bottom-panel shows the corresponding energy boost distribution for these particles. [Right] Same as for the Left case, though here the particles propagated through the turbulent magnetic field setup described in the section 2, which we label "field". The vertical dashed lines in the figures indicate the acceleration frequency obtained for different rigidity particle groups (different colours). A shear flow with $\Gamma_{\text {jet }}=10$ is considered in each case.

The resultant rigidity dependence of the acceleration time obtained from both the "random" scattering case, and the "field" realised case, are shown in Fig. 4. As demonstrated by the figure, considerably faster acceleration is experienced by low-rigidity particles in the "field" case, than in the reference "random" case.

\section{Conclusions}

Recent observations suggest in situ acceleration of very energetic electrons along the extent of extra-galactic jets. Shear acceleration is a promising candidate mechanism for driving the acceleration. In this work, we consider the limitations of simple scattering models in the context of physically plausible field configurations. In particular, we focus on the topological constraints introduced by requiring a solenoidal field in the vicinity of a shear discontinuity and conclude that such a description may provide an inaccurate picture of the acceleration process.

Eschewing the widely adopted decomposition of synthetic turbulent fields into Fourier plane waves, we present a naturally cylindrical solenoidal field formulation as a superposition of Fourier- 


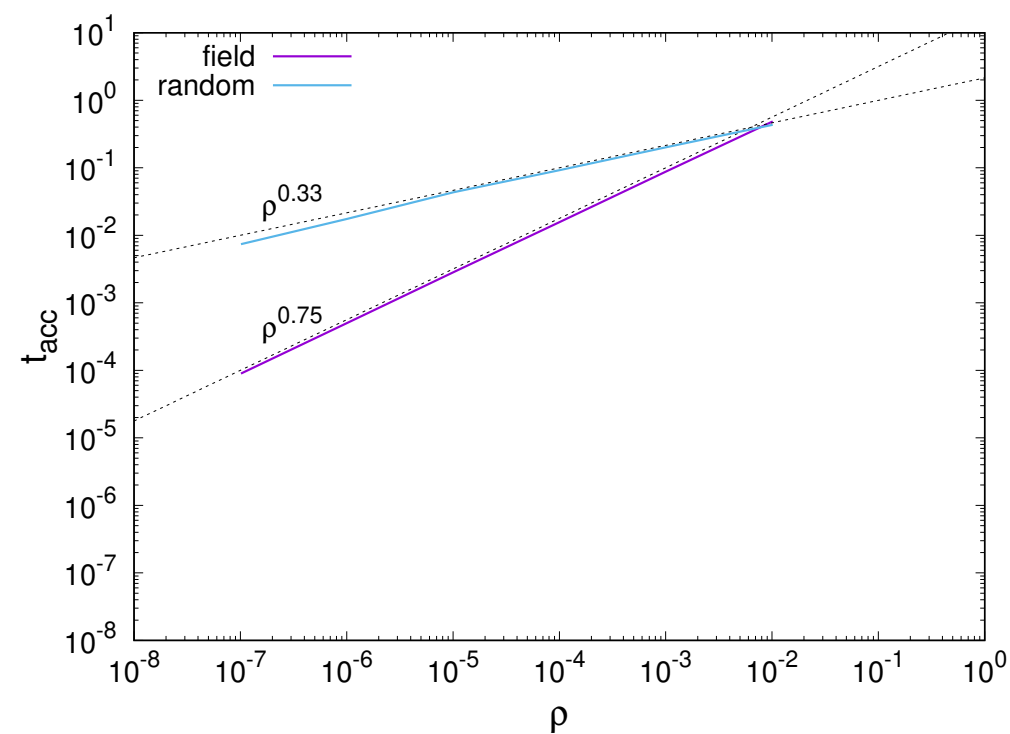

Figure 4: The rigidity (energy) dependence of the acceleration time for both the "random" and "field" cases. Time is in units of $r_{\text {jet }} / c$.

Bessel-type modes. A consequence of this physically motivated field description is an attenuation of the power from large to small scales with decreasing distance from the discontinuity. Simulations of particle motion in the field support the conclusion that low energy particles will be accelerated significantly more efficiently than predicted by simple scattering models. Our results highlight the necessity for consideration of the detailed magnetic field topology in and around the jet boundaries.

\section{References}

[1] S. Jester, D.E. Harris, H.L. Marshall and K. Meisenheimer, New Chandra Observations of the Jet in $3 C$ 273. I. Softer X-Ray than Radio Spectra and the X-Ray Emission Mechanism, ApJ 648 (2006) 900 [astro-ph/0605529].

[2] H. Abdalla et al., Resolving acceleration to very high energies along the jet of Centaurus A, Nature 582 (2020) 356 [2007. 04823].

[3] F.M. Rieger, An Introduction to Particle Acceleration in Shearing Flows, Galaxies 7 (2019) 78 [1909.07237].

[4] G.M. Webb, The Diffusion Approximation and Transport Theory for Cosmic Rays in Relativistic Flows, ApJ 340 (1989) 1112.

[5] A. Achterberg, Y.A. Gallant, J.G. Kirk and A.W. Guthmann, Particle acceleration by ultrarelativistic shocks: theory and simulations, MNRAS 328 (2001) 393 [astro-ph/0107530].

[6] M. Ostrowski, Diffusive acceleration of cosmic ray particles at tangential discontinuity of velocity field, A\&A 238 (1990) 435. 
[7] J. Giacalone and J.R. Jokipii, The transport of cosmic rays across a turbulent magnetic field, The Astrophysical Journal 520 (1999) 204.

[8] G. Benford and R. Protheroe, Fossil agn jets as ultrahigh-energy particle accelerators, Monthly Notices of the Royal Astronomical Society 383 (2008) 663.

[9] D. Mukherjee, G. Bodo, A. Mignone, P. Rossi and B. Vaidya, Simulating the dynamics and non-thermal emission of relativistic magnetized jets I. Dynamics, MNRAS 499 (2020) 681 [2009.10475].

[10] S. Komissarov and O. Porth, Numerical simulations of jets, New A Rev. 92 (2021) 101610.

[11] H. Biglari, P. Diamond and P. Terry, Influence of sheared poloidal rotation on edge turbulence, Physics of Fluids B: Plasma Physics 2 (1990) 1.

[12] K.-C. Shaing, E. Crume Jr and W. Houlberg, Bifurcation of poloidal rotation and suppression of turbulent fluctuations: A model for the l-h transition in tokamaks, Physics of Fluids B: Plasma Physics 2 (1990) 1492.

[13] Y. Zhang and S. Mahajan, Edge turbulence scaling with shear flow, Physics of Fluids B: Plasma Physics 4 (1992) 1385.

[14] J.-L. Vay, Simulation of beams or plasmas crossing at relativistic velocity, Physics of Plasmas 15 (2008) 056701.

\section{Acknowledgments}

The authors gratefully acknowledge computing resources and support by the MPIK staff. The authors wish to acknowledge the Irish Centre for High-End Computing (ICHEC) for the provision of computational facilities and support. 